\title{
Emulador de Lazo Abierto para Turbinas Eólicas de Paso Fijo
}

\author{
Fernando Martínez, Santiago de Pablo y Luis C. Herrero \\ Universidad de Valladolid, Escuela de Ingenierías Industriales, Departamento de Tecnología \\ Electrónica, Calle Francisco Mendizábal 1, 47014 Valladolid-España (e-mail: \\ fer_mart@tele.uva.es)
}

Recibido Jun. 23, 2010; Aceptado Ago. 06, 2010; Versión Final recibida Oct. 06, 2010

\section{Resumen}

Se presenta un nuevo emulador para turbinas eólicas de paso fijo, que permite realizar pruebas de laboratorio de los sistemas de control de los generadores asociados a las turbinas eólicas. La novedad del emulador consiste en utilizar un esquema en lazo abierto. El emulador consiste en el acoplamiento en serie de los siguientes elementos: una fuente de tensión de CC, una resistencia de potencia y un motor de CC. La modificación de la tensión en CC tiene el mismo efecto que la variación de la velocidad del viento en una turbina eólica. Se incluyen: 1) los fundamentos teóricos del emulador, 2) la realización de simulaciones utilizando las aplicaciones informáticas Excel y Matlab/Simulink, y 3) los resultados experimentales obtenidos en una bancada de máquinas eléctricas. Se comprueba que el emulador permite reproducir las curvas de potencia mecánica de una turbina de paso fijo, aunque con ciertas limitaciones a baja velocidad de giro.

Palabras clave: energías renovables, emulador de turbina eólica, turbinas eólicas, generador eólico

\section{Open Loop Emulator for Fixed Pitch Wind Turbines}

\begin{abstract}
A novel emulator for fixed pitch wind turbines that allows laboratory testing of the generator control systems associated with the turbines is presented. The novelty of the emulator is to be an open loop scheme. The emulator consists of the coupling in series of a source of DC voltage, a power resistance and a DC motor. The modification of the input DC voltage has the same effect as the variation of wind speed in a fixed pitch wind turbine. This article includes: 1) theoretical foundations of the emulator, 2) Excel and Simulink simulations, and 3) experimental results obtained in a bench of electrical machines. It is shown that the emulator can reproduce the mechanical power curves of a fixed pitch turbine, but with certain limitations at low speed.
\end{abstract}

Keywords: renewable energy, wind turbine emulator, wind turbine, wind generator 


\section{INTRODUCCIÓN}

Las turbinas eólicas de paso fijo se han utilizado durante mucho tiempo en los sistemas de generación de energía, para baja potencia y potencias media. Hoy en día, las turbinas de potencia media y alta utilizan palas de paso variable, mientras que la utilización de turbinas de paso fijo se limita a las aplicaciones de baja potencia. Las turbinas eólicas emplean sistemas de control de potencia para adaptarse a los cambios de velocidad del viento, cuyo objetivo es extraer tanta potencia como sea posible del viento. En las turbinas de gran tamaño, la potencia extraída del viento se controla mediante la velocidad de la turbina y mediante el ángulo de paso de las palas. Cuando el tamaño de las turbinas es menor, la potencia se controla únicamente por medio de uno de los dos sistemas: hay turbinas que controlan la velocidad, pero no el ángulo de paso (López y Vannier, 2009), y otras que pueden variar el ángulo de paso, pero no la velocidad (Kazmierkowski et al., 2002).

Las curvas de potencia mecánica de una turbina de paso fijo pueden expresarse como una función de la velocidad del viento, como se muestra en la figura 1 (Pena et al., 1995; Brune et al., 1994), donde pu significa "per unit". Para cada velocidad del viento, la curva de potencia tiene un valor máximo que se produce a una velocidad diferente. Un emulador de turbina eólica debe ser capaz de reproducir estas curvas de potencia.

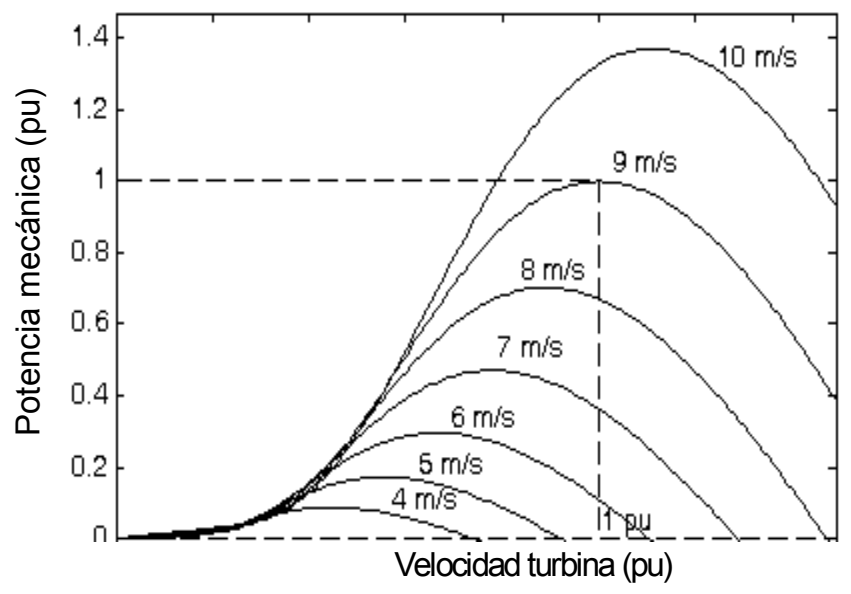

Fig. 1: Curvas de potencia mecánica de la turbina eólica para diferentes valores de la velocidad del viento.

En las turbinas de velocidad variable, la velocidad de giro se controla mediante un convertidor electrónico de potencia (Muyeen et al., 2010). Éste tiene una doble misión, además de controlar la velocidad, se encarga de convertir la tensión alterna producida por el generador en corriente continua. Un segundo convertidor se encarga de la transformación de la tensión continua en alterna para ser inyectada a la red.

Cuando se diseña un convertidor electrónico de potencia para una aplicación de energía eólica, después de realizar las simulaciones correspondientes (Melício y Mendes, 2007), es necesario realizar las pruebas de laboratorio pertinentes, antes de hacer las pruebas finales de campo. En la fase intermedia de pruebas de laboratorio, se requiere algún tipo de sistema que emule el funcionamiento de la turbina eólica. Los diseñadores de accionamientos electrónicos para aerogeneradores suelen emular las turbinas eólicas mediante motores de corriente continua, motores de inducción de jaula de ardilla o de motores de imanes permanentes. En todos los casos, el motor se controla para que tenga unas curvas de potencia similares a las de una turbina eólica. Se utiliza un control basado en microprocesador, que toma como variables de entrada la velocidad del viento y la velocidad de la turbina y, a partir de ellos, proporciona el par de referencia del motor que simula la turbina.

El estado de la técnica de los sistemas de emulación de turbinas eólicas, es tal que el motor de $\mathrm{CC}$ es el más frecuentemente utilizado. En este caso se utiliza un sistema de control (figura 2), 
donde las variables de entrada son la velocidad y el par del motor. El par motor se puede medir, pero en la mayoría de los casos se calcula a partir de la corriente del motor. Estas variables de entrada se introducen en un microprocesador que tiene registradas en su memoria las curvas de la turbina, y cuya salida es la referencia de par del motor de CC. A continuación se indican algunos ejemplos de realizaciones prácticas de este tipo de emuladores. En Bhowmik et al. (1999), la emulación de la turbina se consigue a través de un motor de corriente continua, alimentado por un convertidor de tiristores. A partir de la velocidad de rotación y la velocidad del viento, se calcula el par mecánico de la turbina, que se introduce como referencia al accionamiento del motor de corriente continua, que tiene un sensor de par. En Chinchilla et al. (2004), se utiliza un accionamiento comercial de un motor de CC con control de par. Las características de par de la turbina, en función de la velocidad del viento y la velocidad de la turbina, se almacenan en una memoria. Este par se utiliza como referencia de par del accionamiento del motor de CC. En Monfared et al. (2008) y en Battaiotto et al. (1996), el par motor se calcula a partir de la medida de la corriente, y se introduce en el controlador para emular las curvas de potencia de la turbina. En Cárdenas y Peña (2004), la velocidad del motor CC se calcula y se envía al sistema de control del motor de corriente continua; se utiliza una tabla para almacenar las curvas de coeficiente de potencia frente al ratio de velocidad de la punta de la pala (“tip-speed-ratio", TSR) CP-TSR en el microprocesador.

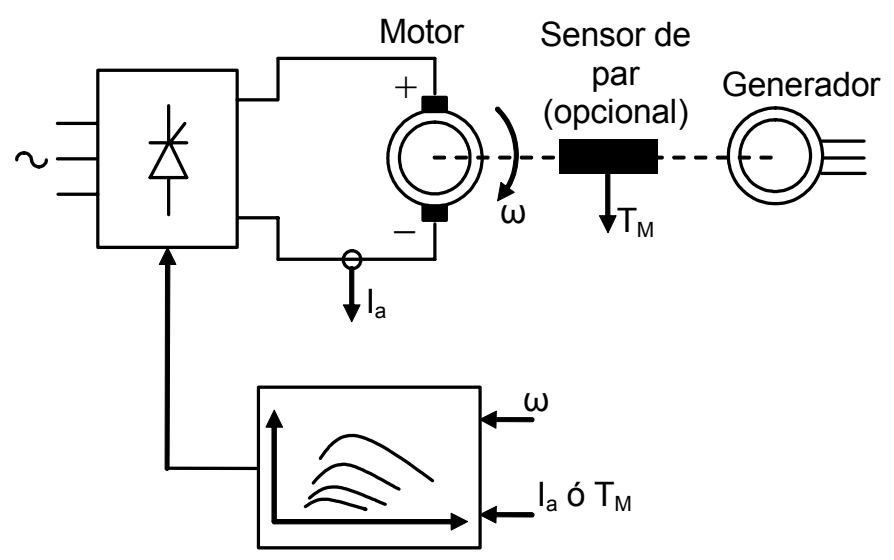

Fig. 2: Esquema de control de un emulador de turbina que emplea un motor de CC.

En los sistemas de emulación se emplean también motores de corriente alterna. En ellos, el sistema de control (figura 3), establece la referencia de par del motor a partir de la velocidad del motor y de la velocidad del viento. El sistema de control del motor de CA se encarga de conseguir que el par del motor sea igual al de referencia. A continuación se incluyen algunos ejemplos de emuladores de turbinas realizados con motores de CA. En Dolan y Lehn (2005), se emplea un motor de imanes permanentes con control vectorial para emular el par mecánico de la turbina. Para la realimentación se emplea un sensor de par en el eje. El control se realiza a través del PC y las señales de disparo se generan en una FPGA (del inglés Field Programmable Gate Array). Tiene en cuenta los cambios en la velocidad del viento desde la base hasta la parte superior del rotor de la turbina, y la sombra de la torre de la turbina. En Kojabadi et al. (2004) y en Munteanu et al. (2005), se utiliza un motor de inducción como emulador de la turbina. A partir de la velocidad del viento y de la velocidad de la turbina, se obtiene el par de la turbina, que se introduce como referencia al regulador del motor de inducción.

En este artículo se presenta un nuevo tipo de emulador de turbina eólica, cuya novedad consiste en trabajar en lazo abierto. No precisa de un sistema realimentado para proporcionar unas curvas de potencia similares a las de la turbina eólica (figura 1). Se presentarán las ecuaciones que demuestran el comportamiento similar a la turbina que pretende emular, junto con las simulaciones y las pruebas experimentales que avalan su utilidad para la función de emulación. 


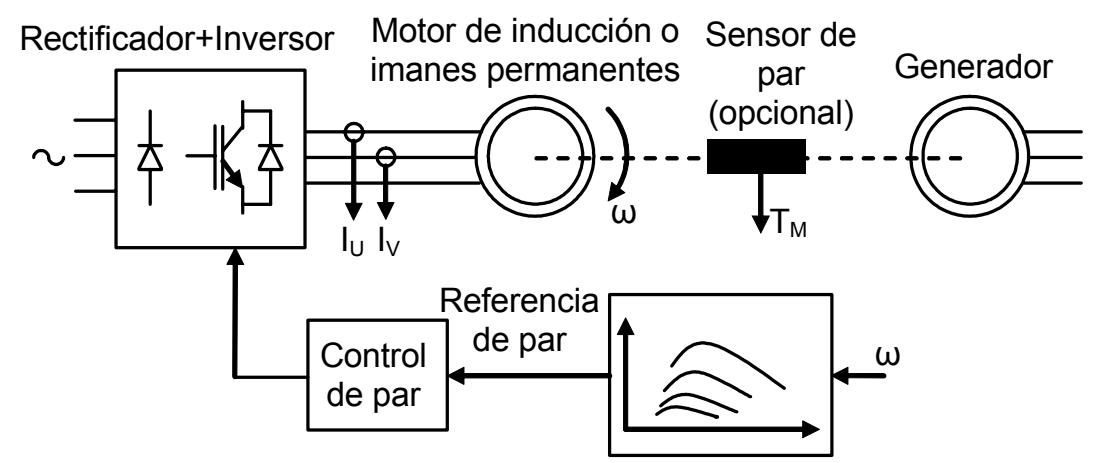

Fig. 3: Esquema de un emulador de turbina que emplea un motor de CA.

\section{METODOLOGÍA}

En primer lugar, se presenta el esquema del emulador propuesto junto con las ecuaciones de funcionamiento que permiten demostrar que sus curvas de potencia tienen forma de parábola invertida, lo que es similar a las curvas de potencia de una turbina de paso fijo, para una velocidad de viento constante. Mediante Excel, se hace una representación inicial de las ecuaciones del emulador de la turbina, en las que se observa que la variación de las curvas de potencia del emulador que se produce al modificar la tensión de entrada en CC, es similar a la variación de las curvas de potencia de la turbina eólica cuando se modifica la velocidad del viento (figura 1). También mediante Excel, se hace una simulación de la potencia en la resistencia serie del emulador, y se puede observar de forma preliminar que hay una limitación de la velocidad inferior del emulador debido al incremento de la potencia en dicha resistencia.

Una vez realizada una simulación elemental, mediante Excel, de las ecuaciones del emulador, se hace una simulación más cercana a la realidad mediante Simulink, utilizando un modelo del motor más cercano a la realidad, como corresponde a los modelos de las librerías de dicho programa comercial de simulación. Se obtienen las curvas de potencia del emulador para diferentes valores de la tensión de entrada en CC y se llega a la conclusión de que son similares a las que se habían obtenido mediante las ecuaciones básicas.

Por último, se presentan las pruebas experimentales, obtenidas mediante una bancada de motores de $440 \mathrm{~W}$, que consta de dos máquinas de CC con excitación independiente y de una dinamo tacométrica, todas ellas acopladas por el eje. Una de las máquinas de CC se empleará como motor, formando parte del emulador de la turbina, mientras que la otra máquina de CC se emplea como generador. Para controlar las máquinas eléctricas se emplean rectificadores semicontrolados. A partir de los datos obtenidos, se generan las curvas de potencia del emulador y se ratifican los resultados de las simulaciones, que indicaban que las dichas curvas eran similares a las de una turbina eólica de paso fijo.

\section{RESULTADOS}

\section{Emulador propuesto}

Se propone una forma de resolver el problema de emular las curvas de potencia mecánica de la turbina eólica, para la realización de pruebas de laboratorio. El emulador se compone de un motor de corriente continua controlado por el inducido, una resistencia $\mathrm{R}$ y una fuente de alimentación de CC de tensión variable, todos ellos conectados en serie (figura 4). La variación de la tensión de CC producirá en el emulador un efecto similar al que produce el cambio de velocidad del viento en una turbina eólica. 


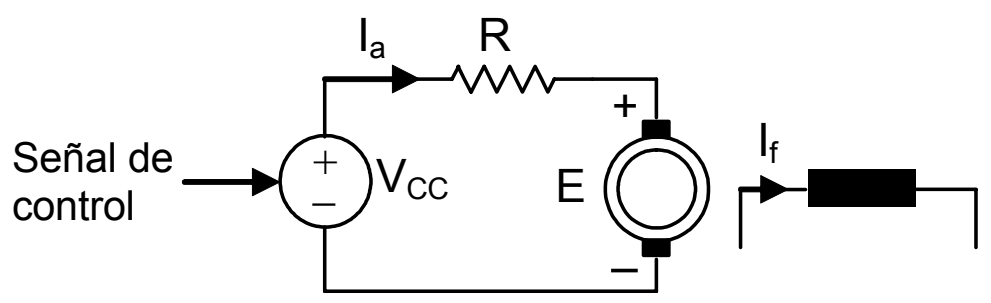

Fig. 4: Diagrama eléctrico del emulador de la turbina eólica.

Como es conocido, las ecuaciones del motor de CC con excitación independiente son:

$$
\begin{aligned}
& E=K_{E} \cdot \omega \\
& K_{E}=L_{a f} \cdot I_{f} \\
& T_{E}=K_{T} \cdot I_{a}
\end{aligned}
$$

La potencia mecánica del motor, despreciando las pérdidas en la resistencia del estator y las pérdidas por rozamiento, es:

$$
P_{m}=E \cdot I_{a}=E\left(\frac{V_{C C}-E}{R}\right)=\frac{K_{E} \cdot \omega \cdot V_{C C}-\left(K_{E} \cdot \omega\right)^{2}}{R}
$$

Para que la potencia mecánica del motor tenga forma de parábola invertida, el valor del coeficiente de tensión $\mathrm{K}_{\mathrm{E}}$ debe ser constante. Para ello, la corriente de excitación $\mathrm{I}_{\mathrm{f}}$ será constante y la excitación del motor debe ser independiente. La representación gráfica de de la ecuación de potencia del motor (4), para varios valores de la tensión en $\mathrm{CC}$, se muestra en la figura 5 donde se puede ver la equivalencia con las curvas de la turbina eólica (figura 1). Los cálculos han empleado un motor de potencia nominal $440 \mathrm{~W}$ y una resistencia serie de $50 \Omega$.

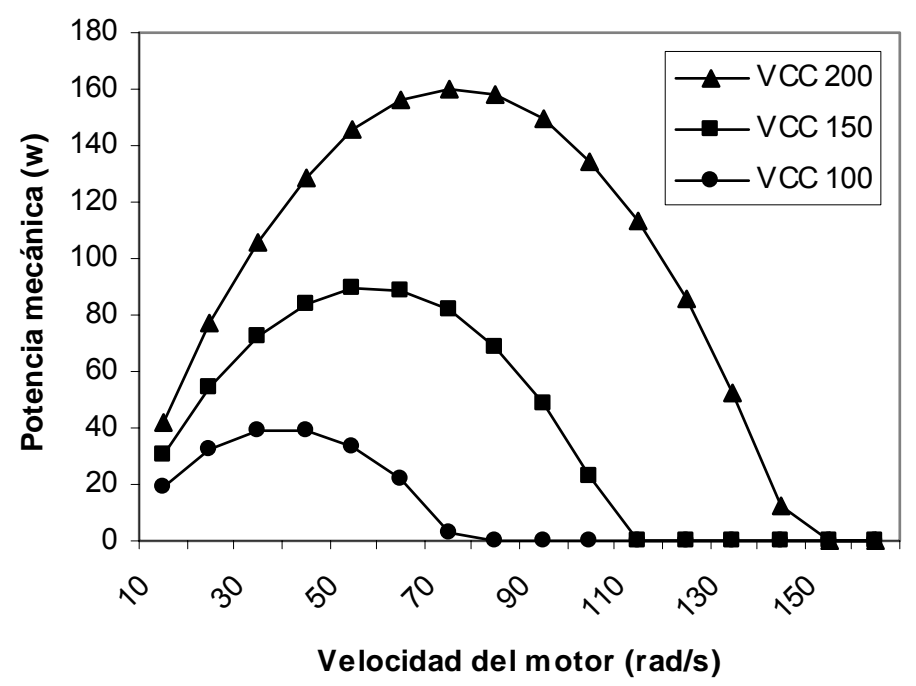

Fig. 5: Curvas de potencia del emulador, calculadas mediante Excel, para diferentes valores de la tensión de la fuente de CC.

Cuando la velocidad del motor es baja, la potencia en la resistencia aumenta significativamente, tomando el siguiente valor:

$$
P_{R}=I_{a}{ }^{2} \cdot R=\left(\frac{V_{C C}-E}{R}\right)^{2} R=\frac{\left(V_{C C}-K_{E} \cdot \omega\right)^{2}}{R}
$$


En la figura 6, se ha representado la potencia en la resistencia serie, empleando los mismos datos que en la figura 5. Se puede observar que la potencia en la resistencia aumenta en gran medida cuando la velocidad es baja. Esto supone una limitación del sistema de emulación, y obliga a limitar la velocidad mínima del emulador.

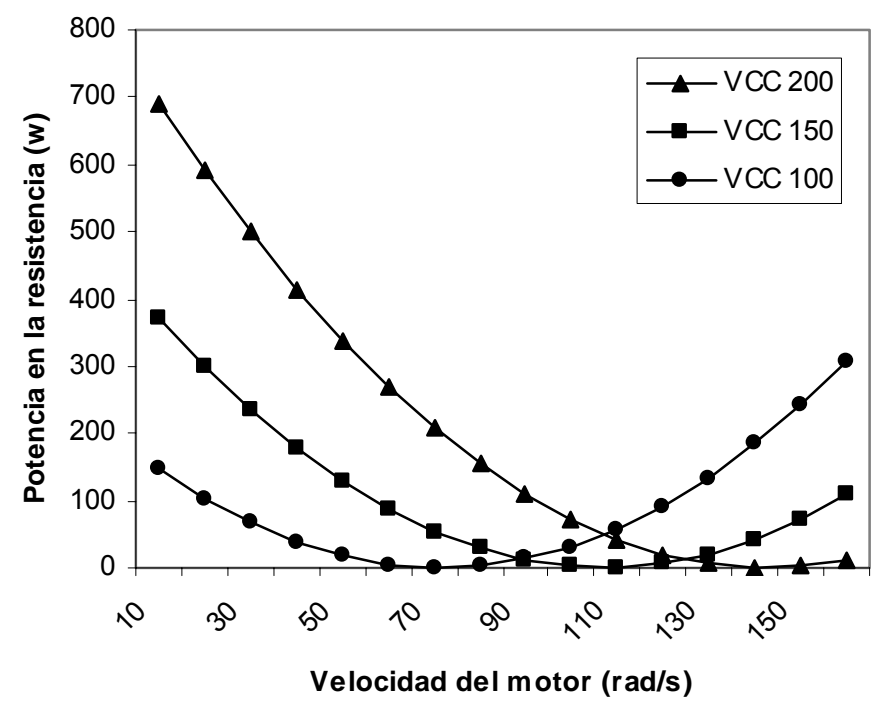

Fig. 6: Cálculo de la potencia en la resistencia serie, realizado mediante Excel, para diferentes valores de la tensión de alimentación en CC.

\section{Resultados de simulación}

Se ha simulado el emulador de turbina eólica, mediante Simulink, para verificar que las curvas de potencia mecánica son similares a los de una turbina eólica de paso fijo. Se ha elegido un motor de $3,75 \mathrm{~kW}$ de las librerías de Simulink. El esquema de simulación (figura 7) tiene los elementos del emulador: tensión de CC, resistencia serie y motor de CC con excitación independiente. Para obtener las curvas de potencia frente a la velocidad de giro, se establece la velocidad del motor mediante una señal en rampa. La velocidad de variación de esta rampa ha de ser muy baja para que la medida de potencia no incluya las variaciones de energía cinética del motor. Las curvas de potencia del motor de CC, para diversos valores de la fuente de tensión de CC, se pueden observar en la figura 8 , para una resistencia serie $R=5 \Omega$. Para cada valor de la tensión de la fuente de $\mathrm{CC}$, hay una curva de potencia mecánica del motor, de forma similar al caso de una turbina eólica. La tensión del emulador se puede considerar equivalente a la velocidad del viento en el caso de una turbina eólica real.

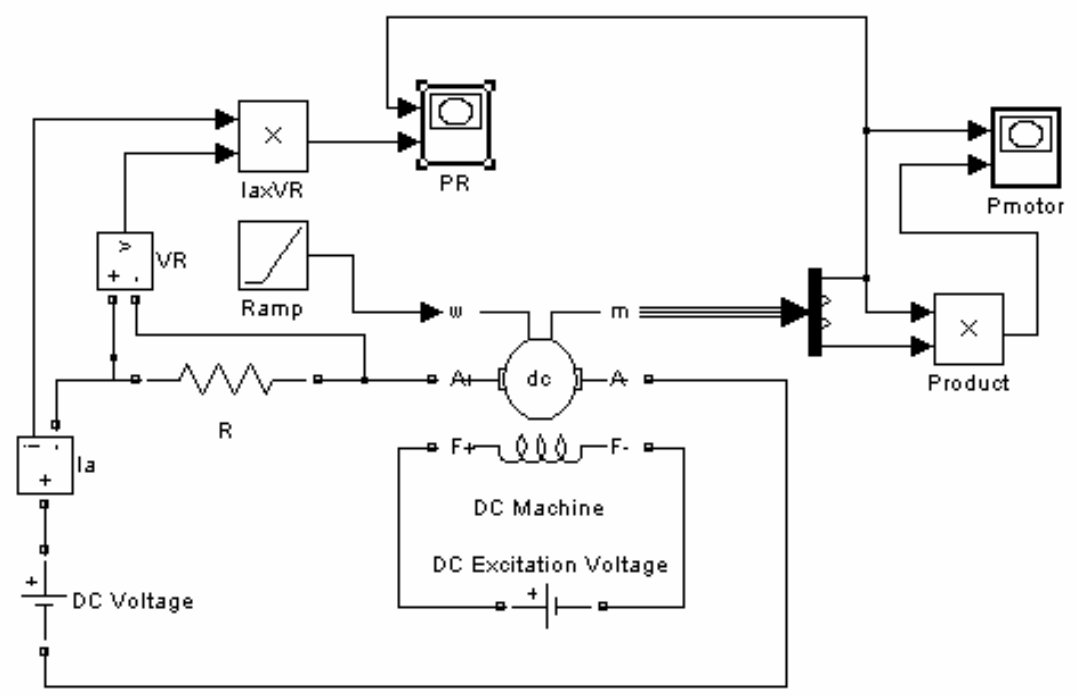

Fig. 7: Esquema del emulador de la turbina simulado mediante Simulink. 


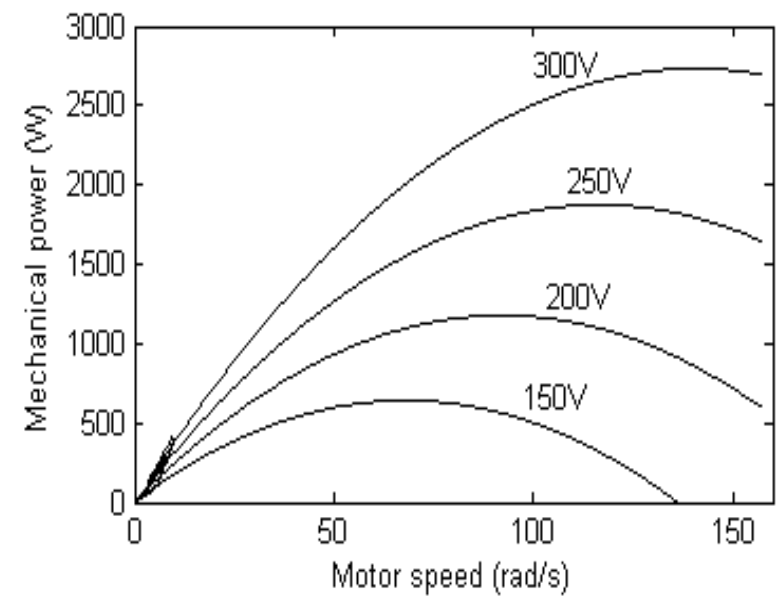

Fig. 8: Curvas de potencia del emulador de la turbina, obtenidas mediante Simulink, para varios valores de fuente de tensión de CC.

\section{Resultados experimentales}

Para la obtención de resultados experimentales se ha empleado un conjunto formado por dos máquinas de CC y una dinamo tacométrica. Una máquina de CC se ha utilizado como motor de CC y la otra se ha empleado como generador de CC (figura 9). La tensión en CC para alimentar al motor se obtiene de un rectificador monofásico semicontrolado. La velocidad del generador se ha controlado mediante otro rectificador del mismo tipo, pero trabajando en modo inversor. El objetivo del experimento es obtener las curvas de potencia mecánica del emulador de la turbina, en función de la velocidad de giro, para varios valores de la tensión de alimentación en CC.

Los parámetros de los elementos empleados son: $R=50 \Omega$, Pmotor $=440 \mathrm{~W}$, Vmotor $=220 \mathrm{~V}$ y $R_{M}$ $=9 \Omega$. Las características del motor de CC y del generador de CC son iguales.

La potencia mecánica del motor se ha calculado como la diferencia entre la potencia eléctrica y las pérdidas en la resistencia interna, según se expresa en la siguiente fórmula,

$$
P_{m}=E \cdot I_{a}-R_{M} \cdot I_{a}^{2}
$$

El acoplamiento de las dos máquinas eléctricas se ha llevado a cabo de forma suave, para evitar un exceso de corriente. Para ello, en primer lugar se ha fijado a cero la tensión del generador $\mathrm{V}_{\mathrm{CC2}}, \mathrm{y}$, después, se ha conectado el rectificador que alimenta el motor.

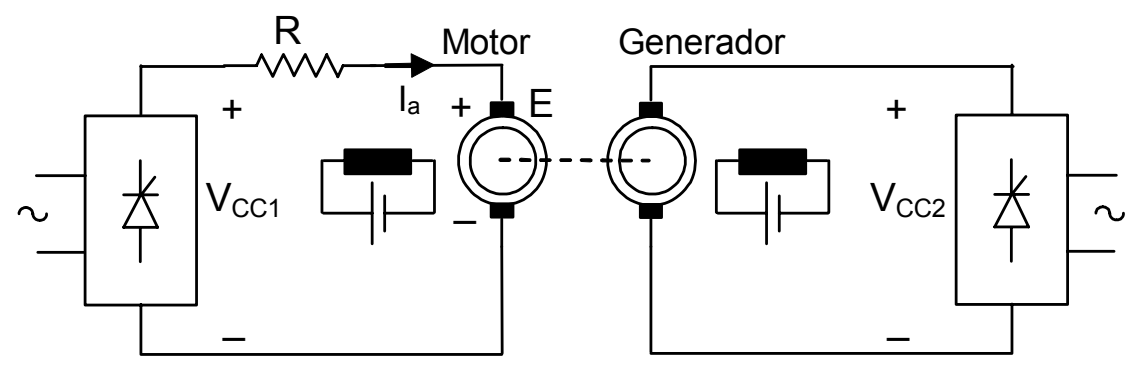

Fig. 9: Esquema empleado en las pruebas experimentales del emulador de la turbina.

Los resultados experimentales se muestran en la figura 10. La tensión del motor $\mathrm{V}_{\mathrm{CC} 1}$ se controla mediante el ángulo de disparo del rectificador. Para cada valor de $\mathrm{V}_{\mathrm{cc} 1}$, la variación de velocidad se controla por medio de la tensión del rectificador del generador $\mathrm{V}_{\mathrm{CC} 2}$ (que trabaja en modo inversor). 


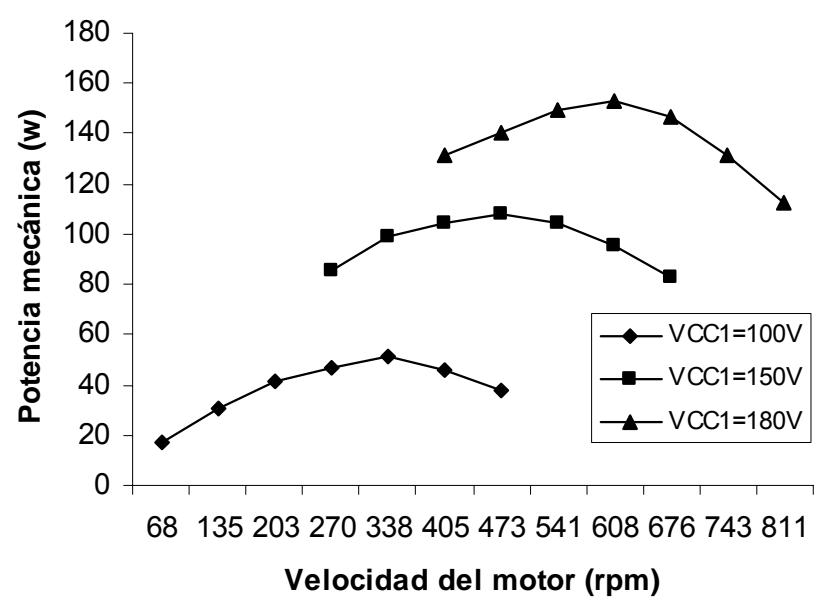

Fig. 10: Potencia mecánica del emulador, obtenida mediante test de laboratorio.

La tensión $V_{c c 1}$ se debe ajustar para cada velocidad, debido a que este valor se ve afectado por la velocidad del motor. El motivo es que la fuerza electromotriz es proporcional a la velocidad del motor.

En la figura 11, se presenta la potencia en la resistencia serie. Se puede ver que su valor aumenta mucho a baja velocidad, lo que supone una limitación de funcionamiento para el emulador propuesto. Para evitar que la potencia en la resistencia tome un valor excesivamente elevado, se necesita limitar la reducción de velocidad, fundamentalmente para altas valores de la tensión del motor.

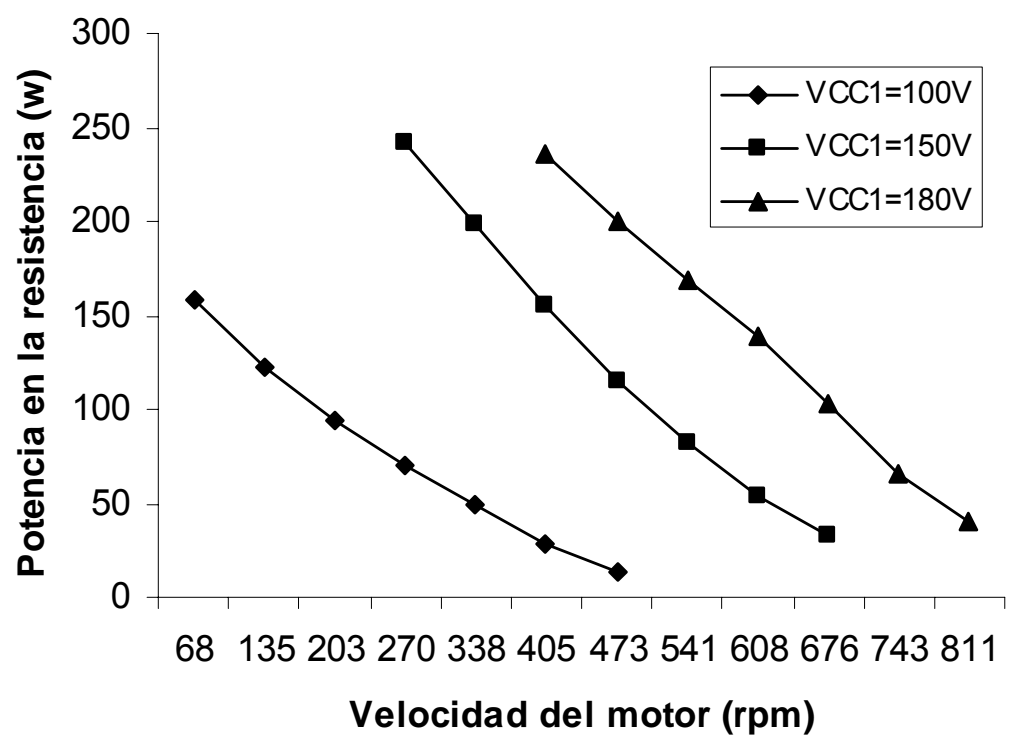

Fig. 11: Potencia en la resistencia del emulador, obtenida mediante pruebas de laboratorio.

\section{DISCUSIÓN}

Se puede observar que las curvas de potencia del emulador propuesto (figuras 5,8 y 10 ) son similares a las curvas de potencia de una turbina eólica de paso fijo (figura 1), donde la variación de la velocidad del viento de la turbina real se puede emular mediante la variación de la tensión en CC de alimentación del emulador. Esta equivalencia consiste en que la forma de las curvas es, en ambos casos, una parábola invertida, con un máximo de potencia que se produce a una velocidad mayor cuando la tensión en CC (emulador) o la velocidad del viento (turbina real) aumenta.

La diferencia de este emulador con el estado de la técnica es que este emulador trabaja en lazo abierto, mientras que los emuladores que se emplean actualmente trabajan en lazo cerrado. El nuevo emulador tiene, intrínsecamente, unas curvas de potencia (figuras 5,8 y 10) similares a las 
de una turbina eólica (figura 1), por lo que no es necesaria la presencia de un sistema de retroalimentación. El estado de la técnica utiliza motores de varios tipos (corriente continua, jaula de ardilla, imanes permanentes), que trabajan de manera intrínseca a velocidad constante, y que sólo mediante la introducción de un sistema para controlar el par y la velocidad puede reproducir las curvas de potencia de la turbina. Al trabajar en lazo abierto, el emulador propuesto es un sistema más simple, que no precisa de un sistema de control del motor, y que, por tanto, no tiene ningún problema de interacción entre los dos bucles de control (emulador de turbina y generador). La patente de este emulador está en proceso.

En los resultados de simulación y experimentales, se observa que la potencia en la resistencia serie tiene un valor similar a la potencia mecánica disponible en el emulador de la turbina, siempre y cuando se trabaje con una velocidad que no sea excesivamente baja, ya que en este caso la potencia se eleva en gran medida (figuras 6 y 11). Por ello, cuando la potencia que se quiere obtener del emulador es elevada, hay que poner un límite a la reducción de velocidad, para evitar daños en la resistencia serie y en el motor, debido al incremento de la corriente. Esto supone unas limitaciones que no tienen los emuladores de lazo cerrado, en los que el sistema de control se encarga de reducir la corriente del motor a baja velocidad.

Por todo ello, se puede afirmar que el emulador propuesto se puede emplear para la realización de pruebas de laboratorio de los sistemas de control de los generadores que van acoplados al eje de una turbina eólica de paso fijo, aunque con ciertas limitaciones a baja velocidad de giro.

\section{CONCLUSIONES}

A partir de la revisión de las referencias citadas, de los cálculos teóricos realizados y de las simulaciones y pruebas experimentales realizadas, se pueden extraer las siguientes conclusiones:

i) se ha presentado un nuevo sistema para la emulación de turbinas eólicas de paso fijo, compuesto por la interconexión en serie de una fuente de tensión variable de CC, una resistencia de potencia y un motor de CC con excitación independiente;

ii) se ha demostrado, mediante desarrollos teóricos, simulaciones y pruebas experimentales, que el emulador puede reproducir las curvas de potencia de una turbina eólica;

iii) el emulador presentado es útil para la realización de pruebas de laboratorio de los convertidores de potencia empleados para controlar el generador de la turbina eólica;

iv) la novedad del emulador consiste en trabajar en lazo abierto, mientras que los emuladores actuales trabajan en lazo cerrado, por lo que el emulador propuesto tiene una estructura intrínseca más parecida a las turbinas eólicas;

v) el emulador propuesto tiene limitaciones a baja velocidad debido al incremento de la potencia en la resistencia serie.

\section{LISTA DE SÍMBOLOS}

$E=$ fuerza electromotriz del motor

$\mathrm{I}_{\mathrm{a}}=$ corriente del estator

$\mathrm{I}_{\mathrm{f}}=$ corriente de excitación

$I_{U}, I_{V}=$ corriente de las fases 1 y 2

$\mathrm{K}_{\mathrm{E}}=$ coeficiente de tensión

$\mathrm{K}_{\mathrm{T}}=$ coeficiente de par

$\mathrm{L}_{\mathrm{af}}=$ inductancia mutua entre excitación y estator

$\mathrm{R}=$ resistencia serie del emulador

$\mathrm{R}_{\mathrm{M}}=$ resistencia interna del motor

$T_{E}=$ par electromagnético del motor

$\mathrm{T}_{\mathrm{M}}=$ par mecánico del motor 
$\mathrm{V}_{\mathrm{CC}}=$ tensión de alimentación en corriente continua

$\omega=$ velocidad del motor

\section{AGRADECIMIENTOS}

Este trabajo ha sido financiado por el Ministerio de Ciencia e Innovación y Fondos FEDER en el Plan Nacional de I+D+I (2004-2007), con la referencia ENE2007-67417.

\section{REFERENCIAS}

Battaiotto P.E., R.J. Mantz y P.F. Puleston, A Wind Turbine Emulator based on a Dual DSP Processor System, Control Eng. Practice: 4(9), 1261-1266 (1996).

Bhowmik, S., R. Spee y H.R. Johan, Performance Optimization for Doubly Fed Wind Power Generation Systems, IEEE Transactions on Industry Applications: 35(4), 949-958 (1999).

Brune, C.S., R. Spée y A.K. Wallace, Experimental evaluation of a variable-speed, double-fed wind power generation system, IEEE Transactions on Industry Applications: 30(3), 648-655 (1994).

Cárdenas, R. y R. Peña, Sensorless Vector Control of Induction Machines for Variable-Speed Wind Energy Applications, IEEE Transactions on Energy Conversion: 19(1), 196-205 (2004).

Chinchilla, M., S. Arnaltes y J.L. Rodriguez-Amenedo, Laboratory set-up for Wind Turbine Emulation, Actas del IEEE International Conference on Industrial Technology (ICIT'04), 553-559, Hammamet, Túnez, 8 al 10 de Diciembre (2004).

Dolan, D.S.L. y P.W. Lehn, Real-Time Wind Turbine Emulator Suitable for Power Quality and Dynamic Control Studies, Actas del International Conference on Power Systems Transients (IPST'05), 1-6, Montreal, Canada, 19 al 23 de Junio (2005).

Kazmierkowski, M.P., R. Krishnan y F. Blaabjerg, Control in Power Electronics Selected Problems, Academic Press, San Diego, USA, 2002.

Kojabadi, H.M., L. Chang y T. Boutot, Development of a Novel Wind Turbine Simulator for Wind Energy Conversion Systems Using an Inverter-Controlled Induction Motor, IEEE Transactions on Energy Conversion: 19(3), 547-552 (2004).

López, M. y J.C. Vannier, Stand-Alone Wind Energy Conversion System with Maximum Power Transfer Control, Ingeniare. Revista Chilena de Ingeniería: 17(3), 329-336 (2009).

Melício, R. y V.M.F. Mendes, Simulación de Convertidores de Potencia en Sistemas Eólicos, Información Tecnológica: 18(4), 25-34 (2007).

Monfared, M., H.M. Kojabadi y H. Rastegar, Static and dynamic wind turbine simulator using a converter controlled dc motor, Renewable Energy: 33(5), 906-913 (2008).

Munteanu, I., N.A. Cutululis, A.I. Bratcu y E. Changa, Optimization of Variable Speed Wind Power Systems Based on a LQG Approach, Control Engineering Practice: 13(7), 903-912 (2005).

Muyeen, S.M., R. Takahashi, T. Murata y J. Tamura, A Variable Speed Wind Turbine Control Strategy to Meet Wind Farm Grid Code Requirements, IEEE Transactions on Power Systems: 25(1), $331-340$ (2010).

Pena, R.S., J.C. Clare y G.M. Asher, Implementation of vector control strategies for a variable speed double-fed induction machine for wind generation system, Actas del 6th European Conference on Power Electronics and Applications (EPE'95), 3075-3080, Sevilla, España, 19 al 21 de Septiembre (1995). 